XX Міжнародний симпозіум «Методи дискретних особливостей в задачах математичної фізики/Discrete Singularities Methods in Mathematical Physics»,

УДК 539.3

MSC 74B20

\title{
Nonlinear Problem of Interface Crack Behavior under the Action of Shearing Wave
}

\author{
A.V. Menshykov ${ }^{1}$, V.A. Menshykov ${ }^{2}$, O.Yu. Kladova ${ }^{2}$ \\ ${ }^{1}$ Centre for Micro- and Nanomechanics (CEMINACS), School of Engineering, University of Ab- \\ erdeen, AB24 3UE Aberdeen, Scotland, UK \\ E-mail: o.menshykov@abdn.ac.uk \\ ${ }^{2}$ National aerospace university named by N.Ye. Zhukovsky \\ «Kharkov aviation Institute», Chkalova str., 17, Kharkiv, Ukraine \\ E-mail:v.menshykov@ukr.net.ua
}

\begin{abstract}
Solution of the problem for an interface crack under the action of a harmonic shear wave is presented. It is shown that the same problems solutions of other authors were performed without taking into account the crack faces contact, and results obtained indicate the interpenetration of the faces, that is not possible. Thus, it is proved that the problem is nonlinear because the positions and sizes of the contact zone are unknown and variable during the loading. The solution is obtained by the boundary integral equations method taking into account the contact interaction of the crack faces: using the Somigliana dynamic identity and the boundary equations arising from them, the transition from the twodimensional problem to the equivalent problem at the boundaries of the domain is realized; the vector components in the boundary integral equations are presented by Fourier series, to prevent the interpenetration of the crack faces and the emergence of tensile forces in the contact zone the Signorini unilateral constraints are involved. The numerical solution is performed by the method of boundary elements with constant approximation of the problem parameters on an element. Numerical researches of the shear wave frequency influence onto the crack faces and adjoining surface displacements, opening and extent of crack faces contact zone are carried out. The quantitative difference between the maximum tangential and normal components of adhesion line and the crack faces displacements is shown. It is shown that the position and length of the contact area change during the load period, and the magnitudes of the contact forces vary along the crack length.
\end{abstract}

Key words: Interface Crack, Harmonic Shear Wave, Boundary Integral Equations, Contact Crack Problem, Contact Forces.

\section{Нелинейная задача о межматериальной трещине под воздействием волны сдвига}

\author{
А.В. Меньшиков ${ }^{1}$, В.А. Меньшиков ${ }^{2}$ О.Ю. Кладова ${ }^{2}$ \\ ${ }^{1}$ Centre for Micro- and Nanomechanics (CEMINACS), School of Engineering, University of Ab- \\ erdeen, AB24 3UE Aberdeen, Scotland, UK \\ E-mail: o.menshykov@abdn.ac.uk \\ ${ }^{2}$ Национальный аэрокосмический университет им. Н.Е. Жуковского \\ «Харьковский авиационный институт», ул. Чкалова, 17, Харьков, Украина \\ E-mail:v.menshykov@ukr.net.ua
}

() Меньшиков А.В., Меньшиков В.А., Кладова О.Ю., 2021 


\begin{abstract}
Представлены материалы решение задачи о межфазной трещине под действием гармонической волны сдвига. Существовавшие ранее решения подобной задачи выполнены без учета контакта берегов, и, как следствие, получены результаты, свидетельствующие о взаимопроникновении берегов, что в действительности невозможно. Сделан вывод о нелинейности задачи, обусловленной тем, что положение и размеры зон контакта берегов неизвестны и переменны во времени. Решение получено методом граничных интегральных уравнений с учетом контактного взаимодействия берегов трещины: используя соотношения Сомильяны и предельные равенства, вытекающие из них, выполнен переход от задачи в двумерной области к эквивалентной задаче на границах области, компоненты векторов сил и перемещений в граничных интегральных уравнениях представлены рядами Фурье, для исключения взаимопроникновения берегов и возникновения растягивающих усилий в зоне контакта привлечены односторонние ограничения Синьорини. Численное решение математической модели выполнено методом граничных элементов с постоянной аппроксимацией параметров задачи на элементе. Проведены численные исследования особенностей смещения берегов трещины и прилегающей к ней интерфейсной поверхности при изменении частоты волны сдвига, исследования зависимости величины раскрытия и протяженности зоны контакта берегов от частоты нагрузки. Показана количественная разница между максимальными касательными и нормальными компонентами перемещений линии сцепления материалов и на берегах трещины. Продемонстрировано, что положение и длина участка контакта берегов изменяются в течение периода нагружения, а величины контактных сил переменны по длине трещины.
\end{abstract}

Ключевые слова: межфазная трещина, гармоническая волна сдвига, метод граничных элементов, зона контакта берегов, контактные силы.

\title{
1. Введение
}

Результаты численного решения задачи о межфазной трещине под действием статической сдвигающей нагрузки, изложены в работах [1-3].

Попытка численного исследования параметров напряженно-деформированного состояния биматериала с межфазной трещиной без учета контакта берегов при нагружении волной сдвига предпринята в работе [4]. Полученные в ней результаты указывают на взаимное проникновение противоположных берегов, что в действительности нереализуемо. Последнее свидетельствует о необходимости учитывать налегание берегов межматериальной трещины, что возможно при нелинейной постановке задачи $[5,6]$.

Представляемые в настоящей работе результаты решения нелинейной задачи теории упругости для биматериала с межфазной трещиной основаны на методе граничных интегральных уравнений (ГИУ) [7, 8] и методе исследования нелинейных динамических задач механики трещин с учетом контактного взаимодействия их берегов $[6,8]$.

\section{2.Формулировка задачи}

Метод ГИУ позволяет установить зависимость между значениями искомых функций внутри рассматриваемой области и их значениями на границе путем перехода от дифференциальных уравнений к следующим из них интегральным соотношениям. Последнее дает нам возможность понизить геометрическую размерность задачи на единицу, а значит уменьшить объем вычислений.

Итак, первый этап использования метода ГИУ состоит в получении интегральных соотношений. Рассмотрим в двумерном пространстве плоскую деформацию 
XX Міжнародний симпозіум «Методи дискретних особливостей в задачах математичної фізики/Discrete Singularities Methods in Mathematical Physics»,

\section{МДОЗМФ/DSMМPh-2021}

двух линейно-упругих полуплоскостей $x_{2} \geq 0, x_{2} \leq 0$ с разными механическими характеристиками (параметрами Ламе $\lambda, \mu$ и плотностями $\rho$ ), которые жестко сцеплены между собой вдоль линии $x_{2}=0$ везде, за исключением промежутка $-1<x_{1}<1$, который представляет собой межматериальную трещину. Перпендикулярно линии сцепления материалов распространяется гармоническая волна сдвига с циклической частотой $\omega=2 \pi / T$, где $T$ - период колебаний. Под действием динамического нагружения противоположные берега трещины деформируются, что приводит к их раскрытию и налеганию с возникновением зон контакта и сил контактного взаимодействия.

Напряженно-деформированное состояние каждой из полуплоскостей описывается уравнениями линейной динамической теории упругости в перемещениях

$$
\begin{aligned}
\left(\lambda^{(m)}+\mu^{(m)}\right) \operatorname{grad} \operatorname{div} \mathbf{u}^{(m)} & (\mathbf{x}, t)+\mu^{(m)} \Delta \mathbf{u}^{(m)}(\mathbf{x}, t)= \\
& =\rho^{(m)} \partial_{t}^{2} \mathbf{u}^{(m)}(\mathbf{x}, t), \mathbf{x} \in \Omega^{(m)}, \quad t \in[0, \infty),
\end{aligned}
$$

где $\mathbf{u}^{(m)}(\mathbf{x}, t)$ - вектор перемещений, $\mathrm{m}=1,2 ; \quad \Delta$ - оператор Лапласа;

со следующими граничными условиями:

- на линии сцепления материалов выполняются условия полного механического контакта, заключающиеся в непрерывности перемещений и напряжений;

- на берегах трещины усилия представляет собой суперпозицию сил от падающей волны и возникающих контактных сил на заранее неизвестных участках налегания берегов.

Подчеркнем, что рассматриваемая задача является нелинейной, поскольку неизвестны положение и размеры зон контакта берегов, изменяющиеся в течение нагружения, неизвестны и усилия на берегах, ибо неизвестны контактные силы.

Перейдем от задачи в двумерной области к эквивалентной задаче на границах области, используя соотношения Сомильяны и вытекающие из них граничные равенства для перемещений и усилий $[6,8]$. В рассматриваемом случае гармонического нагружения граничные интегральные уравнения имеют вид [6]:

$$
\begin{gathered}
-\int_{\Gamma^{(1) \mathrm{cr}}} \tilde{p}_{i}^{k,(1)}(\mathbf{y}) U_{i j}^{(1)}\left(\mathbf{x}, \mathbf{y}, \omega_{k}\right) d \mathbf{y}=-\frac{1}{2} u_{i}^{k,(1)}(\mathbf{x})-\int_{\Gamma^{(1) \mathrm{cr}}} u_{i}^{k,(1)}(\mathbf{y}) W_{i j}^{(1)}\left(\mathbf{x}, \mathbf{y}, \omega_{k}\right) d \mathbf{y}+ \\
+\int_{\Gamma^{*}} u_{i}^{k, *}(\mathbf{y}) W_{i j}^{(1)}\left(\mathbf{x}, \mathbf{y}, \omega_{k}\right) d \mathbf{y}-\int_{\Gamma^{*}} p_{i}^{k, *}(\mathbf{y}) U_{i j}^{(1)}\left(\mathbf{x}, \mathbf{y}, \omega_{k}\right) d \mathbf{y}, \quad \mathbf{x} \in \Gamma^{(1) \mathrm{cr}}, \\
-\int_{\Gamma^{(2) \mathrm{cr}}} \tilde{p}_{i}^{k,(2)}(\mathbf{y}) U_{i j}^{(2)}\left(\mathbf{x}, \mathbf{y}, \omega_{k}\right) d \mathbf{y}=-\frac{1}{2} u_{i}^{k,(2)}(\mathbf{x})-\int_{\Gamma^{(2) \mathrm{cr}}} u_{i}^{k,(2)}(\mathbf{y}) W_{i j}^{(2)}\left(\mathbf{x}, \mathbf{y}, \omega_{k}\right) d \mathbf{y}- \\
-\int_{\Gamma^{*}} u_{i}^{k, *}(\mathbf{y}) W_{i j}^{(2)}\left(\mathbf{x}, \mathbf{y}, \omega_{k}\right) d \mathbf{y}+\int_{\Gamma^{*}} p_{i}^{k, *}(\mathbf{y}) U_{i j}^{(2)}\left(\mathbf{x}, \mathbf{y}, \omega_{k}\right) d \mathbf{y}, \quad \mathbf{x} \in \Gamma^{(2) \mathrm{cr}},
\end{gathered}
$$




$$
\begin{aligned}
& -\int_{\Gamma^{(1) \mathrm{cr}}} \tilde{p}_{i}^{k,(1)}(\mathbf{y}) U_{i j}^{(1)}\left(\mathbf{x}, \mathbf{y}, \omega_{k}\right) d \mathbf{y}=-\frac{1}{2} u_{i}^{k, *}(\mathbf{x})-\int_{\Gamma^{(1) \mathrm{cr}}} u_{i}^{k,(1)}(\mathbf{y}) W_{i j}^{(1)}\left(\mathbf{x}, \mathbf{y}, \omega_{k}\right) d \mathbf{y}+ \\
& +\int_{\Gamma^{*}} u_{i}^{k, *}(\mathbf{y}) W_{i j}^{(1)}\left(\mathbf{x}, \mathbf{y}, \omega_{k}\right) d \mathbf{y}-\int_{\Gamma^{*}} p_{i}^{k, *}(\mathbf{y}) U_{i j}^{(1)}\left(\mathbf{x}, \mathbf{y}, \omega_{k}\right) d \mathbf{y}, \quad \mathbf{x} \in \Gamma^{*}, \\
& -\int_{\Gamma^{(2) \mathrm{cr}}} \tilde{p}_{i}^{k,(2)}(\mathbf{y}) U_{i j}^{(2)}\left(\mathbf{x}, \mathbf{y}, \omega_{k}\right) d \mathbf{y}=-\frac{1}{2} u_{i}^{k, *}(\mathbf{x})-\int_{\Gamma^{(2) \mathrm{cr}}} u_{i}^{k,(2)}(\mathbf{y}) W_{i j}^{(2)}\left(\mathbf{x}, \mathbf{y}, \omega_{k}\right) d \mathbf{y}- \\
& -\int_{\Gamma^{*}} u_{i}^{k, *}(\mathbf{y}) W_{i j}^{(2)}\left(\mathbf{x}, \mathbf{y}, \omega_{k}\right) d \mathbf{y}+\int_{\Gamma^{*}} p_{i}^{k, *}(\mathbf{y}) U_{i j}^{(2)}\left(\mathbf{x}, \mathbf{y}, \omega_{k}\right) d \mathbf{y}, \quad \mathbf{x} \in \Gamma^{*},
\end{aligned}
$$

где: $\tilde{p}_{i}^{k,(m)}(\mathbf{x}), p_{i}^{k, *}(\mathbf{x}), u_{i}^{k, *}(\mathbf{x})$ и $u_{i}^{k,(m)}(\mathbf{x})$ - коэффициенты Фурье усилий и перемещений на берегах трещины и поверхности сцепления; $U_{i j}^{(m)}\left(\mathbf{x}, \mathbf{y}, \omega_{k}\right)$ и $W_{i j}^{(m)}\left(\mathbf{x}, \mathbf{y}, \omega_{k}\right)-$ фундаментальные решения динамической теории упругости.

Заметим, что представление рядами Фурье компонент векторов сил и перемещений в граничных интегральных уравнениях (1-4) необходимо в силу нелинейности граничных условий задачи, это есть первый этап в применении метода исследования нелинейных динамических задач механики трещин с учетом контактного взаимодействия их берегов.

Вторым этапом является привлечение односторонних ограничений Синьорини - дополнительных условий $[6,8]$, исключающих взаимное проникновение берегов и растягивающие усилия в зонах контакта.

Для приведения компонент векторов контактных сил и перемещений берегов трещины в соответствие с ограничениями Синьорини использовался итерационный алгоритм динамической теории упругости:

- задается начальное распределение нагрузки на берегах трещины от падающей волны и задача решается без учета односторонних ограничений, вычисляются составляющие вектора разрыва перемещений;

- корректируются нормальные составляющие вектора разрыва перемещений так, чтобы удовлетворять односторонним ограничениям;

- вычисляются составляющие вектора контактных сил взаимодействия;

- составляющие вектора контактных сил взаимодействия берегов трещины корректируются, чтобы удовлетворять односторонним ограничениям и закону трения;

- совершается переход к следующему итерационному шагу до достижения требуемой точности решения задачи.

На втором этапе использования метода ГИУ - численное решение системы уравнений (1-4) - использован прямой метод граничных элементов с постоянной аппроксимацией параметров задачи на элементе $[7,8]$. Верхний и нижний берега трещины и линия сцепления материалов аппроксимированы одномерными граничными элементами.

Авторы провели численные исследования, посвященные выявлению особенностей смещений берегов межматериальной трещины и примыкающей к ней интерфейсной поверхности, величины раскрытия берегов и протяженности зоны их контакта, величины возникающих контактных сил в условиях нагружения биматериала волной сдвига. 
XX Міжнародний симпозіум «Методи дискретних особливостей в задачах математичної фізики/Discrete Singularities Methods in Mathematical Physics»,

\section{МДОЗМФ/DSMMPh-2021}

\section{3. Расчетные исследования.}

Расчеты выполнены для трещины-отрезка длины $2 L=2$ вдоль линии соединения материалов сталь - алюминий. Материалы характеризуются следующими параметрами: для стали - модуль упругости $E=207$ ГПа, коэффициент Пуассона $v=0,288$ и плотность $\rho=7860$ кг $/ \mathrm{m}^{3}$; для алюминия - модуль упругости $E=70$ ГПа, коэффициент Пуассона $v=0,347$ и плотность $\rho=2700$ кг $/ \mathrm{M}^{3}$.

Нагрузка на берегах трещины задавалась по закону плоской гармонической волны сдвига, направленной по нормали к линии сцепления материалов. Частота гармонической нагрузки характеризовалось приведенным волновым числом $k_{2} L=\omega L / c_{2}$, здесь $c_{2}$ - скорость поперечных волн в алюминии. Приведенное волновое число в расчетах составляло $k_{2} L=1,0$. Полагалось, что контакт противоположных берегов трещины осуществляется с трением, при этом коэффициент трения задавался равным $k_{\tau}=0,5$.

Исследовались направления и величины смещений берегов межматериальной трещины, протяженность зоны контакта берегов и возникающие контактные силы на берегах трещины.

На рис. 1 представлены результаты расчетных исследований тангенциальных компонент перемещений $\left(\bar{U}_{\tau}=U_{\tau} / L\right)$ берегов межматериальной трещины и участков линии сцепления биматериала примыкающих к вершинам трещины, а именно, распределения максимальных тангенциальных компонент перемещений участков линии сцепления биматериала в сравнении с перемещениями противоположных берегов вдоль длины трещины. Здесь 1 и 2 - перемещения берегов трещины, 3 - перемещения участков линии сцепления стали и алюминия.

На рис. 2 представлены распределения максимальных нормальных компонент перемещений $\left(\bar{U}_{n}=U_{n} / L\right)$ участков линии сцепления биматериала в сравнении с перемещениями противоположных берегов вдоль длины трещины. Здесь 1 и 2 перемещения берегов трещины, 3 - перемещения участков линии сцепления стали и алюминия.

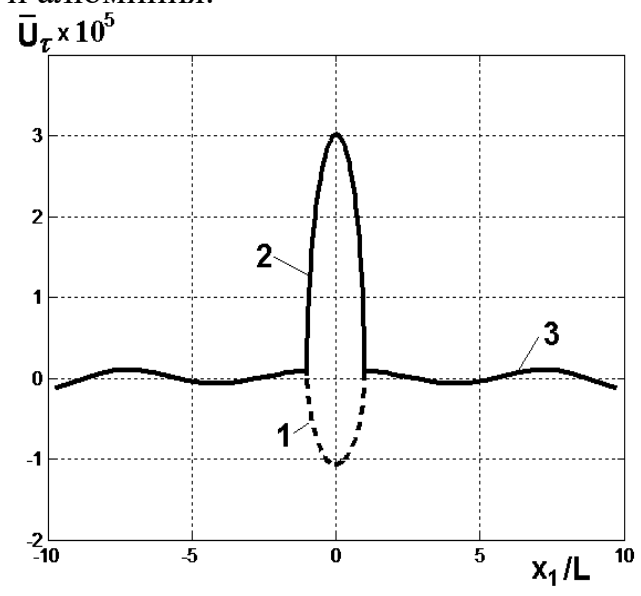

Puc. 1

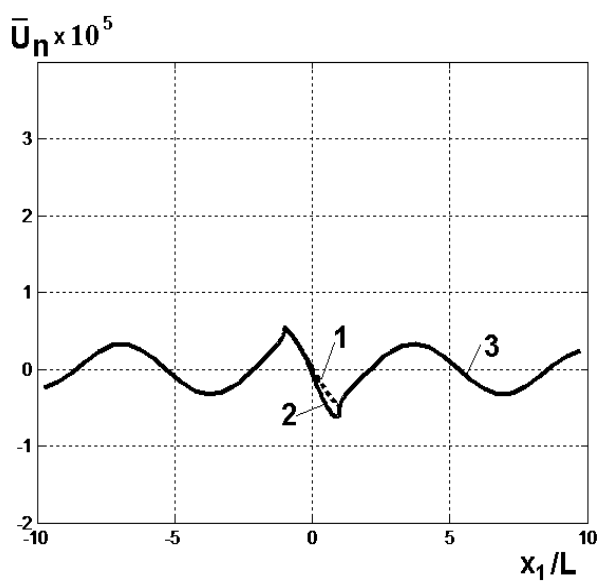

Puc. 2 


\section{4. Заключение}

В результате численного решения нелинейной задачи теории упругости для биматериала с межфазной трещиной при нагружении волной сдвига получены распределения тангенциальных и нормальных компонент смещений берегов трещины и линии сцепления материалов, длины участка контакта берегов, нормальных и тангенциальных компонент контактных сил в течение периода нагружения.

Показано, что:

- величины тангенциальных и нормальных компонент смещения берегов межфазной трещины отличаются на порядок и более высокие значения имеют тангенциальные компоненты;

- величины максимальных тангенциальных компонент перемещения линии сцепления материалов и берегов трещины отличаются на порядок, в то время как величины максимальных нормальных компонент смещения берегов трещины и линии сцепления материалов имеют близкие значения;

- положение и длина участка контакта берегов принципиально меняется в течение периода нагружения;

- величины контактных сил изменяются как по длине трещины, так и в течение периода нагружения.

\section{ЛИТЕРАТУРА}

1. Comninou M. The interface crack. ASME J. Appl. Mech. 1977. V.44. P. 631-636.

2. Comninou M. The interface crack in a shear field. ASME J. Appl. Mech. 1978. V. 45. P. 287-290.

3. Ostrik V.I. Contact of faces of a rectilinear crack under complex loading and various contact conditions. Acta Mech. 2019. 230. P. 3741-3758.

4. Guz A.N., Guz I.A., Men'shikov A.V., Men'shikov V.A. Penny-shaped crack at the interface between elastic half-spaces under the action of a shear wave. Int. Appl. Mech. 2009. 45, N 5. P. 534-539.

5. Guz A.N. Nonclassical Problems of Fracture/Failure Mechanics: On the Occasion of the 50th Anniversary of Research (Review). Int. Appl. Mech. 2019. 55. N 4. P. 343415.

6. Menshykov O.V., Menshykova M.V., Guz I.A. 3-Delastodynamic contact problem for an interface crack under harmonic loading. Engineering Fracture Mechanics. 2012. V. 80. P. 52-59.

7. Crouch S.L., Starfield A.M. Boundary element methods in solid mechanics. LondonBoston-Sidney: George Allen \& Unwin, 1983. 328 p.

8. Guz A.N., Guz I.A., Men'shikov A.V., Men'shikov V.A. Three-Dimensional Problems in the Dynamic Fracture Mechanics of Materials with Interface Cracks (Review). Int. Appl. Mech. 2013. 49, N 1. P. 1-61. 
XX Міжнародний симпозіум «Методи дискретних особливостей в задачах математичної фізики/Discrete Singularities Methods in Mathematical Physics», МДОЗМФ/DSMМPh-2021

\title{
Нелінійна задача про міжматеріальну тріщину під впливом хвилі зсуву
}

\author{
О.В. Меньшиков ${ }^{1}$, В.О. Меньшиков ${ }^{2}$, О.Ю. Кладова ${ }^{2}$ \\ ${ }^{1}$ Centre for Micro- and Nanomechanics (CEMINACS), School of Engineering, University of Ab- \\ erdeen, AB24 3UE Aberdeen, Scotland, UK \\ E-mail: o.menshykov@abdn.ac.uk \\ ${ }^{2}$ Національний аерокосмічний університет ім. М.Є.Жуковського \\ «Харківський авіаційний інститут», вул. Чкалова, 17, Харків, Україна \\ E-mail:v.menshykov@ukr.net.ua
}

Подано матеріали рішення задачі про міжматеріальну тріщину-розріз під дією гармонійної хвилі зсуву. Показано, що попередні рішення подібної задачі було виконано без урахування контакту берегів, $\mathrm{i}$, як наслідок, отримано результати, які свідчать о взаємопроникненні берегів, що у дійсності не можливо. Таким чином доведено що задача э нелінійною оскільки положення та розміри зон контакту берегів невідомі та змінні під час навантаження. Рішення отримано методом граничних інтегральних рівнянь 3 урахуванням контактної взаємодії берегів тріщини: використовуючи співвідношення Сомільяни і граничні рівності, що випливають 3 них, виконано перехід від задачі у двовимірної області до еквівалентної задачі на межах області, компоненти векторів сил та переміщень у граничних інтегральних рівняннях представлено рядами Фур'є, для виключення взаємопроникнення берегів та виникнення зусиль, що розтягають, у зоні контакту залучено односторонні обмеження Сіньоріні. Чисельне рішення математичної моделі виконано методом граничних елементів з постійною апроксимацією параметрів задачі на елементі. Проведені чисельні дослідження особливостей зсувів берегів міжматеріальної тріщини і прилеглої до неї інтерфейсної поверхні при зміні частоти хвилі зсуву, дослідження залежності від частоти навантаження величини розкриття і протягу зони контакту берегів. Показана кількісна різниця між максимальними дотичними і нормальними компонентами переміщень лінії зчеплення матеріалів та на берегах тріщини. Продемонстровано, що положення та довжина ділянки контакту берегів змінюються протягом періоду навантаження, а величини контактних сил перемінні по довжині тріщини.

Ключові слова: міжматеріальна тріщина, гармонійна хвиля зсуву, метод граничних елементів, зона контакту берегів, контактні сили. 